\title{
DISOCCLUSION OF 3D LIDAR POINT CLOUDS USING RANGE IMAGES
}

\author{
P. Biasutti ${ }^{\mathrm{a}, \mathrm{b}}$, J-F. Aujol ${ }^{\mathrm{a}}$, M. Brédif ${ }^{\mathrm{c}}$, A. Bugeau ${ }^{\mathrm{b}}$ \\ ${ }^{\text {a } U n i v e r s i t e ́ ~ d e ~ B o r d e a u x, ~ I M B, ~ C N R S ~ U M R ~ 5251, ~ I N P, ~} 33400$ Talence, France. \\ ${ }^{\mathrm{b}}$ Université de Bordeaux, LaBRI, CNRS UMR 5800, 33400 Talence, France. \\ ${ }^{\mathrm{c}}$ Université Paris-Est, LASTIG MATIS, IGN, ENSG, F-94160 Saint-Mandé, France. \\ \{pierre.biasutti, aurelie.bugeau\}@1abri.fr, jean-francois.aujol@math.u-bordeaux.fr, mathieu.bredif@ign.fr
}

KEY WORDS: LiDAR, MMS, Range Image, Disocclusion, Inpainting, Variational, Segmentation, Point Cloud

\begin{abstract}
:
This paper proposes a novel framework for the disocclusion of mobile objects in 3D LiDAR scenes aquired via street-based Mobile Mapping Systems (MMS). Most of the existing lines of research tackle this problem directly in the 3D space. This work promotes an alternative approach by using a 2D range image representation of the 3D point cloud, taking advantage of the fact that the problem of disocclusion has been intensively studied in the 2D image processing community over the past decade. First, the point cloud is turned into a 2D range image by exploiting the sensor's topology. Using the range image, a semi-automatic segmentation procedure based on depth histograms is performed in order to select the occluding object to be removed. A variational image inpainting technique is then used to reconstruct the area occluded by that object. Finally, the range image is unprojected as a 3D point cloud. Experiments on real data prove the effectiveness of this procedure both in terms of accuracy and speed.
\end{abstract}

\section{INTRODUCTION}

Over the past decade, street-based Mobile Mapping Systems (MMS) have encountered a large success as the onboard 3D sensors are able to map full urban environments with a very high accuracy. These systems are now widely used for various applications from urban surveying to city modeling (Serna and Marcotegui, 2013, Hervieu et al., 2015, El-Halawany et al., 2011, Hervieu and Soheilian, 2013, Goulette et al., 2006). Several systems have been proposed in order to perform these acquisitions. They mostly consist in optical cameras, 3D LiDAR sensor and GPS combined with Inertial Measurement Unit (IMU), built on a vehicle for mobility purposes (Paparoditis et al., 2012, Geiger et al., 2013). They provide multi-modal data that can be merged in several ways, such as lidar point clouds colored by optical images or lidar depth maps aligned with optical images.

Although these systems lead to very complete 3D mapping of urban scenes by capturing optical and 3D details (pavements, walls, trees, etc.), they often acquire mobile objects that are not persistent to the scene. This often happens in urban environments with objects such as cars, pedestrians, traffic cones, etc. As LiDAR sensors cannot penetrate through opaque objects, those mobile objects cast shadows behind them where no point has been acquired (Figure 1, left). Therefore, merging optical data with the point cloud can be ambiguous as the point cloud might represent objects that are not present in the optical image. Moreover, these shadows are also largely visible when the point cloud is not viewed from the original acquisition point of view. This might end up being distracting and confusing for visualization. Thus, the segmentation of mobile objects and the reconstruction of their background remain a strategic issue in order to improve the understability of urban 3D scans. We refer to this problem as disocclusion in the rest of the paper.

In real applicative contexts, we acknowledge that the disoccluded regions might cause a veracity issue for end-users so that the reconstruction masks should be kept in the metadata. Using these masks, further processing steps may then choose to process differently disoccluded regions (e.g. kept for artefact-free visualizations or discarded during object extractions).

We argue that working on simplified representations of the point cloud, especially range images, enables specific problems such as disocclusion to be solved not only using traditional 3D techniques but also using techniques brought by other communities (image processing in our case).

In this work, we aim at presenting a novel framework for the fast and efficient disocclusion of LiDAR point clouds. Our first contribution is to provide a fast segmentation technique for dense and sparse point clouds to extract full objects from the scene by leveraging the implicit range image topology (Figure 1, center). A second contribution is to introduce a fast and efficient variational method for the disocclusion of a point cloud using range image representation while taking advantage of an horizontal prior without any knowledge of the color or texture of the represented objects (Figure 1, right).
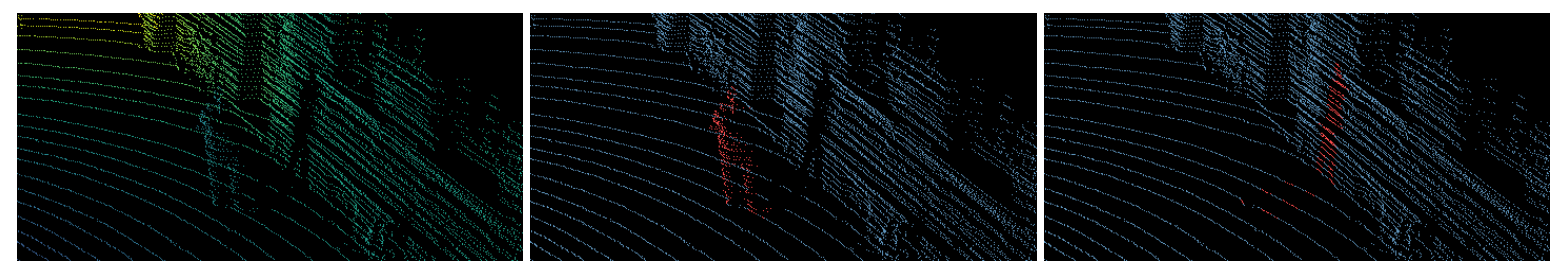

Figure 1. One result of our proposed method. (left) original point cloud, (center) segmentation, (right) disocclusion. 
The paper is organized as follows: after a review on related stateof-the-art, we detail how the point cloud is turned into a range image. In section 3, both segmentation and disocclusion aspects of the framework are explained. We then validate these approaches on different urban LiDAR data. Finally a conclusion and an opening are drawn.

\section{RELATED WORKS}

The growing interest for MMS over the past decade has lead to many works and contributions for solving problems of segmentation and disocclusion. In this part, we present a state-of-the-art on both segmentation and disocclusion.

\subsection{Point cloud segmentation}

The problem of point cloud segmentation has been extensively addressed in the past years. Three types of methods have emerged: geometry-based techniques, statistical techniques and techniques based on simplified representations of the point cloud.

Geometry-based segmentation The first well-known method in this category is region-growing where the point cloud is segmented into various geometric shapes based on the neighboring area of each point (Huang and Menq, 2001). Later, techniques that aim at fitting primitives (cones, spheres, planes, cubes ...) in the point cloud using RANSAC (Schnabel et al., 2007) have been proposed. Others look for smooth surfaces (Rabbani et al., 2006). Although those methods do not need any prior about the number of objects, they often suffer from over-segmenting the scene and as a result objects are segmented in several parts.

Statistical segmentation The methods in this category analyze the point cloud characteristics (Demantke et al., 2011, Weinmann et al., 2015, Brédif et al., 2015). They consider different properties of the PCA of the neighborhood of each point in order to perform a semantic segmentation. It leads to a good separation of points that belongs to static and mobile objects, but not to the distinction between different objects of the same class.

Simplified model for segmentation MMS LiDAR point clouds typically represent massive amounts of unorganized data that are difficult to handle, different segmentation approaches based on a simplified representation of the point cloud have been proposed. (Papon et al., 2013) proposes a method in which the point cloud is first turned into a set of voxels which are then merged using a variant of the SLIC algorithm for super-pixels in 2D images (Achanta et al., 2012). This representation leads to a fast segmentation but it might fail when the scale of the objects in the scene is too different. Another simplified model of the point cloud is presented by (Zhu et al., 2010). The authors take advantage of the implicit topology of the sensor to represent the point cloud as a 2-dimensional range image in order to segment it before performing classification. The segmentation is done through a graph-based method as the notion of neighborhood is easily computable on a 2D image. Although the provided segmentation algorithm is fast, it suffers from the same issues as geometry-based algorithms such as over-segmentation or incoherent segmentation. Moreover, all those categories of segmentation techniques are not able to treat efficiently both dense and sparse LiDAR point clouds e.g. point clouds aquired with high or low sampling rates compared to the real-world feature sizes. In this paper, we present a novel simplified model for segmentation based on histograms of depth in range images.

\subsection{Disocclusion}

Disocclusion of a scene has only been scarcely investigated for 3D point clouds (Sharf et al., 2004, Park et al., 2005, Becker et al., 2009). These methods mostly work on complete point clouds rather than LiDAR point clouds. This task, also referred to as inpainting, has been much more studied in the image processing community. Over the past decades, various approaches have emerged to solve the problem in different manners. Patchbased methods such as (Criminisi et al., 2004) (and more recently (Buyssens et al., 2015b, Lorenzi et al., 2011)) have proven their strengths. They have been extended for RGB-D images (Buyssens et al., 2015a) and to LiDAR point clouds (Doria and Radke, 2012) by considering an implicit topology in the point cloud. Variational approaches represent another type of inpainting algorithms (Chambolle and Pock, 2011, Bredies et al., 2010, Weickert, 1998, Bertalmio et al., 2000). They have been extended to RGB-D images by taking advantage of the bi-modality of the data (Ferstl et al., 2013, Bevilacqua et al., 2017). Even if the results of the disocclusion are quite satisfying, those models require the point cloud to have color information as well as the 3D data. In this work, we introduce an improvement to a variational disocclusion technique by taking advantage of a horizontal prior.

\section{METHODOLOGY}

The main steps of the proposed framework, from the raw point cloud to the final result, are described in Figure 2. We detail each of these steps in this section.

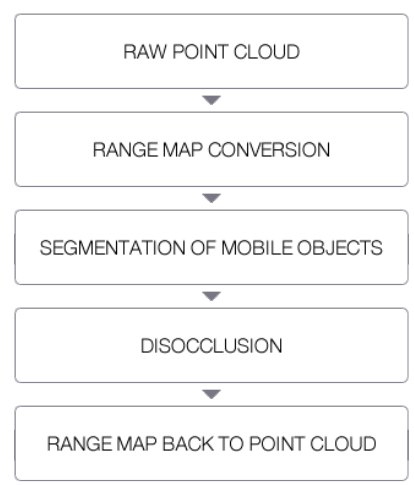

Figure 2. Overview of the proposed framework.

\subsection{Range maps and sensor topology}

The key point of the proposed approach is to work on a simplified representation of the point cloud known as a $2 \mathrm{D}$ range map. The acquired dataset simply consisted in a mapping of the scene, the range map is obtained using the implicit topology of the sensor. The fact that most raw LiDAR acquisitions offer an intrinsic 2D sensor topology is rarely considered. Namely, LiDAR points may obviously be ordered along scanlines, yielding the first dimension of the sensor topology, linking each LiDAR pulse to the immediately preceding and succeeding pulses within the same scanline. For most LiDAR devices, one can also order the consecutive scanlines so as to consider a second dimension of the sensor topology across the scanlines. 2D LiDAR sensors (i.e. : featuring a single simultaneous scanline acquisition) generally send an almost constant number $H$ of pulses per scanline (or per turn for 360 degree 2D LiDARs), so that range measurements may be organized in an image of size $W \times H$, where $W$ is the 


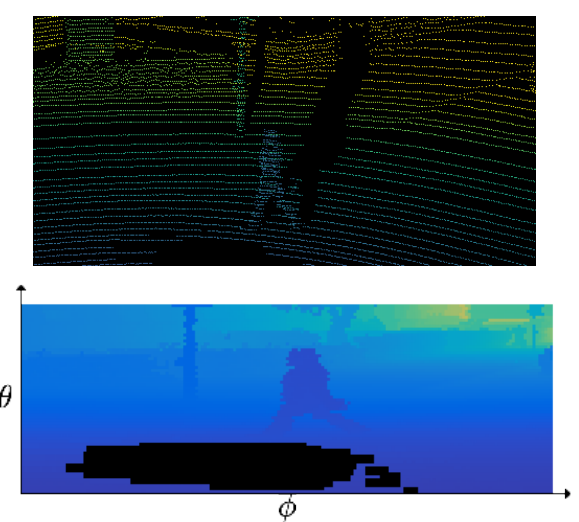

Figure 3. Example of a point cloud from the KITTI database (top) turned into a range image (bottom). Note that the black area in (b) corresponds to pulses with no returns.

number of consecutive scanlines and thus a temporal dimension. 3D LiDAR sensors are based on multiple simultaneous scanline acquisitions (e.g. $H=64$ ) such that scanlines may be stacked horizontally to form an image, as illustrated in Figure 3.

Whereas LiDAR pulses are emitted somewhat regularly, many pulses yield no range measurements due, for instance, to reflective surfaces, absorption or absence of target objects (e.g. in the sky direction). Therefore the sensor topology is only a relevant approximation for emitted pulses but not for echo returns, such that the range image is sparse with undefined values where pulses measured no echoes. Considering multi-echo datasets as a multilayer depth image is beyond the scope of this paper.

The sensor topology only provides an approximation of the immediate 3D point neighborhoods, especially if the sensor moves or turns rapidly compared to its sensing rate. We argue however that this approximation is sufficient for most purposes, as it has the added advantage of providing pulse neighborhoods that are reasonably local both in terms of space and time, thus being robust to misregistrations, and being very efficient to handle (constant time access to neighbors). Moreover, as LiDAR sensor designs evolve to higher sampling rates within and/or across scanlines, the sensor topology will better approximate spatio-temporal neighborhoods, even in the case of mobile acquisitions.

We argue that raw LiDAR datasets generally contain all the information (scanline ordering, pulses with no echo, number of points per turn...) to enable a constant-time access to a well-defined implicit sensor topology. However it sometimes occurs that the dataset received further processings (points were reordered or filtered, or pulses with no return were discarded). Therefore, the sensor topology may only be approximated using auxilliary point attributes (time, $\theta, \phi$, fiber id...) and guesses about acquisition settings (e.g. guessing approximate $\Delta$ time values between successive pulse emissions).

In the following sections, the range image is denoted $u^{R}$.

\subsection{Point cloud segmentation}

We now propose a segmentation technique based on range histograms. For the sake of simplicity, we assume that the ground is relatively flat and remove ground points by plane fitting.

Instead of segmenting the whole range image $u^{R}$ directly, we first split this image in $S$ sub-windows $u_{s}^{R}, s=1 \ldots S$ of size

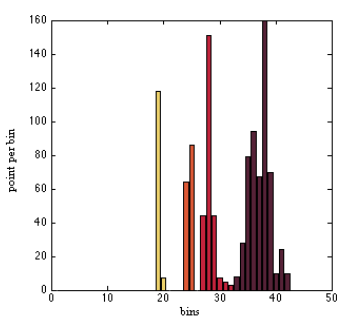

a.

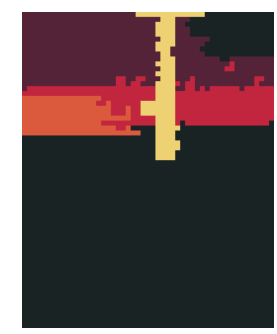

b.
Figure 4. Result of the histogram segmentation using (Delon et al., 2007). (a) segmented histogram (bins of 50cm), (b) result in the range image using the same colors.
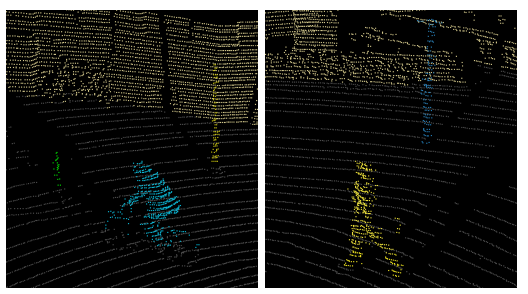

Figure 5. Example of point cloud segmentation using our model on various scenes.

$W_{s} \times H$ along the horizontal axis. For each $u_{s}^{R}$, a depth histogram $h_{s}$ of $B$ bins is built. This histogram is automatically segmented into $C_{s}$ classes using the a-contrario technique presented in (Delon et al., 2007). This technique presents the advantage of segmenting a 1D-histogram without any prior assumption, e.g. the underlying density function or the number of objects. Moreover, it aims at segmenting the histogram following an accurate definition of an admissible segmentation, preventing over and under segmentation from appearing. Examples of segmented histograms are given in Figure 4.

Once the histogram of successive sub-images have been segmented, we merge together the corresponding classes by checking the distance between each of their centroids. Let us define the centroid of the $\mathrm{i}^{t h}$ class $C_{s}^{i}$ in the histogram $h_{s}$ of the sub-image $u_{s}^{R}$ as follows:

$$
\mathcal{C}_{s}^{i}=\frac{\sum_{b \in C_{s}^{i}} b \times h_{s}(b)}{\sum_{b \in C_{s}^{i}} h_{s}(b)}
$$

where $b$ are all bins belonging to class $C_{s}^{i}$. The distance between two classes $C_{s}^{i}$ and $C_{r}^{j}$, of two consecutive windows can be defined as follows:

$$
d\left(C_{s}^{i}, C_{r}^{j}\right)=\left|\mathcal{C}_{s}^{i}-\mathcal{C}_{r}^{j}\right|
$$

Finally, we can set a threshold such that if $d\left(C_{s}^{i}, C_{r}^{j}\right) \leq \tau$, classes $C_{s}^{i}$ and $C_{r}^{j}$ should be merged. Results of this segmentation procedure can be found in Figure 5. We argue that the choice of $W_{s}, B$ and $\tau$ mostly depends on the type of data that is being treated (sparse or dense). For sparse point clouds, $B$ has to remain small (e.g. 50) whereas for dense point clouds, this value can be increased (e.g. 200). In practice, we found out that good segmentations may be obtained on various kind of data by setting $W_{s}=0.5 \times B$ and $\tau=0.2 \times B$. Note that the windows are not required to be overlapping in most cases, but for very sparse point clouds, an overlap of $10 \%$ is enough to reach good segmentation. 


\subsection{Disocclusion}

The segmentation technique introduced above provides masks for the objects that require disocclusion. As mentioned in the beginning, we propose a variational approach to the problem of disocclusion of the point cloud. The Gaussian diffusion algorithm provides a very simple algorithm for the disocclusion of objects in 2D images by solving partial differential equations. This technique is defined as follows:

$$
\left\{\begin{array}{l}
\frac{\partial u}{\partial t}-\Delta u=0 \text { in } \Omega \times(0, T) \\
u(0, x)=u_{0}(x) \text { in } \Omega
\end{array}\right.
$$

having $u$ an image defined on $\Omega, t$ being a time range and $\Delta$ the Laplacian operator. As the diffusion is performed in every direction, the result of this algorithm is often very smooth. Therefore, the result in 3D lacks of coherence as shown in Figure 7.b.

In this work, we assess that the structures that require disocclusion are likely to evolve smoothly along the $x_{W}$ and $y_{W}$ axis of the real world as defined in Figure 6 . Therefore, we set $\vec{\eta}$ for each pixel to be a unitary vector orthogonal to the projection of $z_{W}$ in the $u^{R}$ range image. This vector will define the direction in which the diffusion should be done to respect this prior. Note that most of MLS systems provide georeferenced coordinates of each point that can be used to define $\vec{\eta}$.

We aim at extending the level lines of $u$ along $\vec{\eta}$. This can be expressed as $\langle\nabla u, \vec{\eta}\rangle=0$. Therefore, we define the energy $F(u)=\frac{1}{2}(\langle\nabla u, \vec{\eta}\rangle)^{2}$. The disocclusion is then computed as a solution of the minimization problem $\inf _{u} F(u)$. The gradient of this energy is given by $\nabla F(u)=-\left\langle\left(\nabla^{2} u\right) \vec{\eta}, \vec{\eta}\right\rangle=-u_{\vec{\eta} \vec{\eta}}$, where $u_{\vec{\eta} \vec{\eta}}$ stands for the second order derivative of $u$ with respect to $\vec{\eta}$ and $\nabla^{2} u$ for the Hessian matrix. The minimization of $F$ can be done by gradient descent. If we cast it into a continuous framework, we end up with the following equation to solve our disocclusion problem:

$$
\left\{\begin{array}{l}
\frac{\partial u}{\partial t}-u_{\vec{\eta} \vec{\eta}}=0 \text { in } \Omega \times(0, T) \\
u(0, x)=u_{0}(x) \text { in } \Omega
\end{array}\right.
$$

using previously mentioned notations. We recall that $\Delta u=$ $u_{\vec{\eta} \vec{\eta}}+u_{\vec{\eta}^{T} \vec{\eta}^{T}}$, where $\vec{\eta}^{T}$ stands for a unitary vector orthogonal to $\vec{\eta}$. Thus, Equation (4) can be seen as an adaptation the Gaussian diffusion equation (3) to respect the diffusion prior in the direction $\vec{\eta}$. Figure 7 shows a comparison between the original Gaussian diffusion algorithm and our modification. The Gaussian diffusion leads to an over-smoothing of the scene, creating an aberrant surface whereas our modification provides a result that is more plausible.

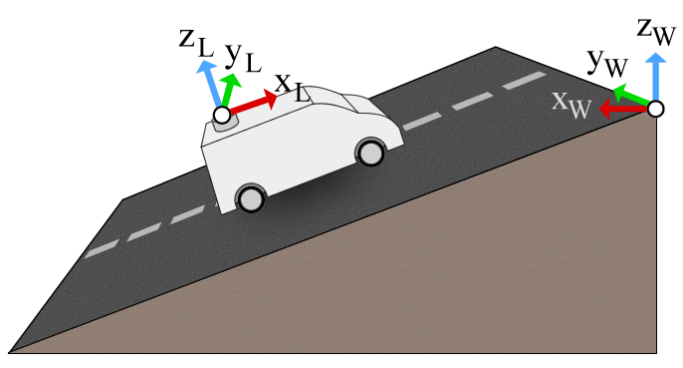

$\mathrm{Z}_{\mathrm{W}}$

Figure 6. Definition of the different frames between the LiDAR sensor $\left(x_{L}, y_{L}, z_{L}\right)$ and the real world $\left(x_{W}, y_{W}, z_{W}\right)$.
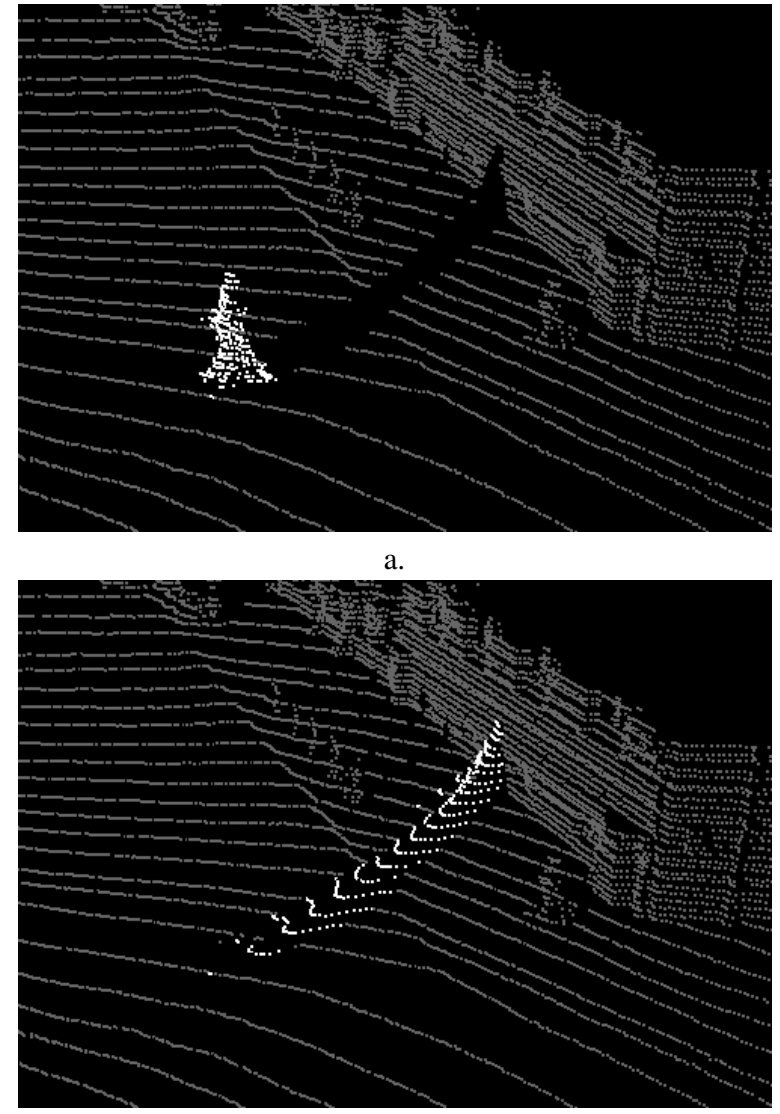

b.

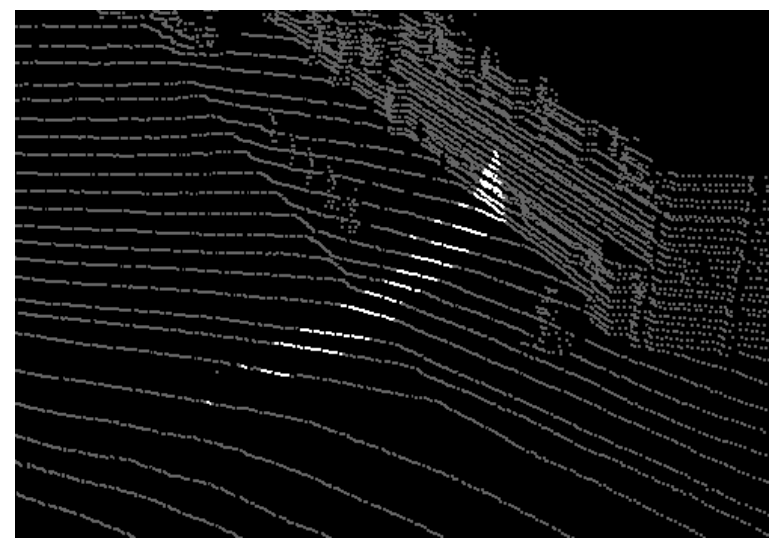

c.

Figure 7. Comparison between disocclusion algorithms. (a) is the original point cloud (white points belong to the object to be disoccluded), (b) the result after Gaussian diffusion and (c) the result with our proposed algorithm. 
The equation proposed in (4) can be solved iteratively. The number of iterations simply depends on the size of the area that needs to be filled in.

\subsection{Range image to 3D point cloud}

After the segmentation and the disocclusion, we need to turn the range image back to the $3 \mathrm{D}$ space. For every point $p_{i}$ of the original point cloud, we define $p_{i}^{o}$ and $p_{i}^{e}$ respectively the point of emission and the point of echo of $p_{i}$. We denote $d_{\text {orig }}\left(p_{i}\right)$ the original range of $p_{i}$ and $d_{\text {rec }}\left(p_{i}\right)$ its range after disocclusion. The new coordinates $p_{i}^{\text {final }}$ of each point can be obtained using the following formula:

$$
p_{i}^{f i n a l}=p_{i}^{o}+\left(p_{i}^{e}-p_{i}^{o}\right) \times \frac{d_{\text {rec }}\left(p_{i}\right)}{d_{\text {orig }}\left(p_{i}\right)}
$$

The range image can then be easily turned back to a $3 \mathrm{D}$ point cloud while including the disocclusion.

\section{RESULTS}

In this part, the results of the segmentation of various objects and the disocclusion of their background are detailed.

\subsection{Sparse point cloud}

A first result is shown in Figure 8. This result is obtained for a sparse point cloud $\left(\approx 10^{6}\right.$ pts) of the KITTI database (Geiger et al., 2013). A pedestrian is segmented out of the scene using our proposed segmentation technique. The segmentation result is used as a mask for the disocclusion of its background using our modified variational technique for disocclusion. Figure 8.a shows the original range image. In Figure 8.b, the dark region corresponds to the result of the segmentation step on the pedestrian. For practical purpose, a very small dilatation is applied to the mask (radius of $2 \mathrm{px}$ in sensor topology) to ensure that no outlier points (near the occluder's silhouette with low accuracy or on the occluder itself) bias the reconstruction. Finally, Figure 8.c shows the range image after the reconstruction. We can see that the disocclusion performs very well as the pedestrian has completely disappeared and the result is visually plausible in the range image.

In this scene, $\vec{\eta}$ has a direction that is very close to the $x$ axis of the range image and the 3D point cloud is acquired using a panoramic sensor. Therefore, the coherence of the reconstruction can be checked by looking how the acquisition lines are connected. Figure 9 shows the reconstruction of the same scene in 3 dimensions. We can see that the acquisition lines are properly retrieved after removing the pedestrian. This result was generated in 4.9 seconds using Matlab on a $2.7 \mathrm{GHz}$ processor. Note that a similar analysis can be done on the results presented in Figure 1.

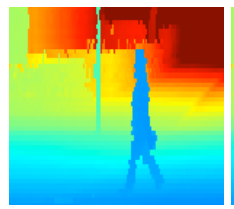

a.

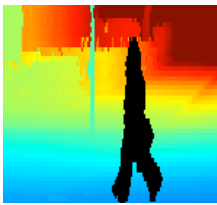

b.

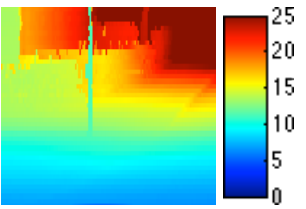

c.
Figure 8. Result of disocclusion on a pedestrian on the KITTI database (Geiger et al., 2013). (a) is the original range image, (b) the segmented pedestrian (dark), (c) the final disocclusion. Depth scale is given in meters.

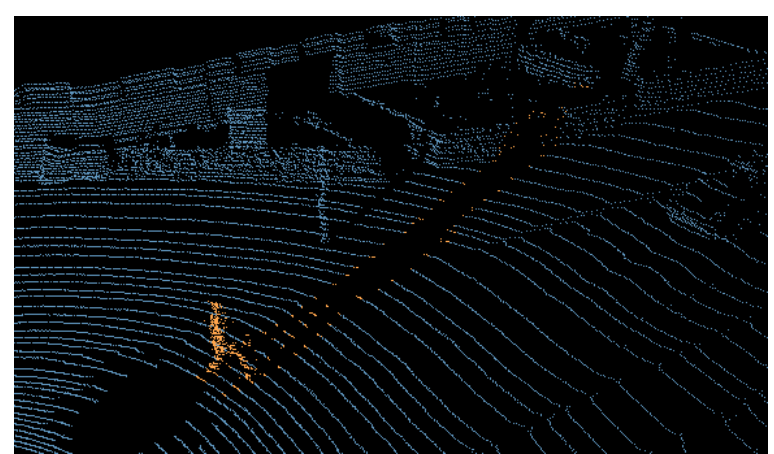

a.

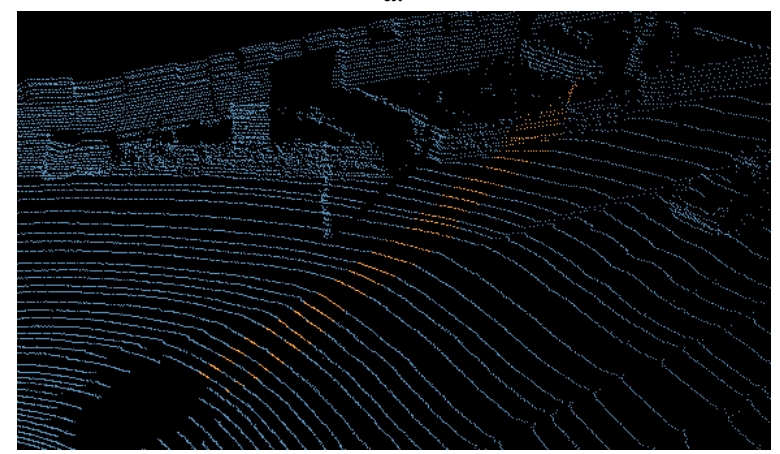

b.

Figure 9. Result of the disocclusion on a pedestrian in 3D. (a) is the original mask highlighted in $3 \mathrm{D}$, (b) is the final reconstruction.

\subsection{Dense point cloud}

In this work, we aim at presenting a model that performs well on both sparse and dense data. Figure 10 shows a result of the disocclusion of a car in a dense point cloud. This point cloud was acquired using the Stereopolis-II system (Paparoditis et al., 2012) and contains over 4.9 million points. In Figure 10.a, the original point cloud is displayed with the color based on the reflectance of the points for a better understanding of the scene. Figure 10.b highlights the segmentation of the car using our model, dilated to prevent aberrant points. Finally, Figure 10.c depicts the result of the disocclusion of the car using our method.

We can note that the car is perfectly removed from the scene. It is replaced by the ground that could not have been measured during the acquisition. Although the reconstruction is satisfying, some gaps are left in the point cloud. Indeed, in the data used for this example, pulse returns with large deviation values were discarded. Therefore, the windows and the roof of the car are not present in the point cloud before and after the reconstruction as no data is available.

\subsection{Quantitative analysis}

To conclude this section, we perform a quantitative analysis of our disocclusion model on the KITTI dataset. The experiment consists in removing areas of various point clouds in order to reconstruct them using our model. Therefore, the original point clouds can serve as ground truths. Note that areas are removed while taking care that no objects are present in those locations. Indeed, this test aims at showing how the disocclusion step behaves when reconstructing backgrounds of objects. The size of the removed areas corresponds to an approximation of a pedestrian's size at 8 meters from the sensor in the range image $(20 \times 20$ px $)$. 


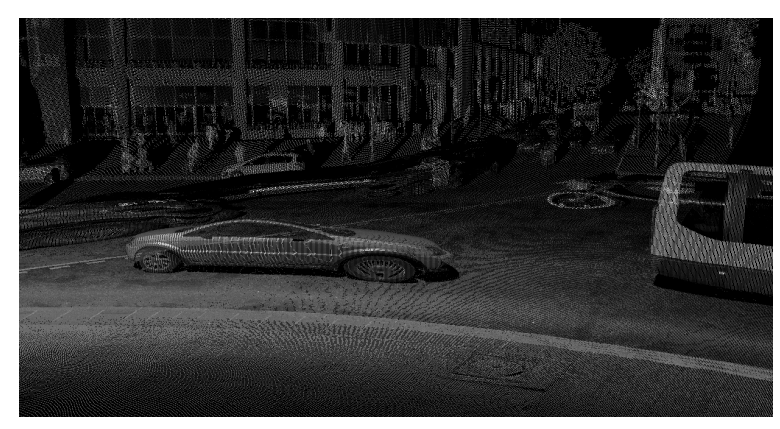

a.

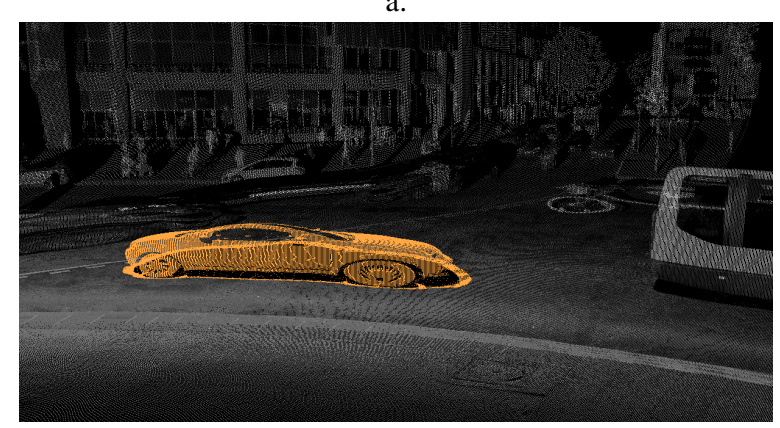

b.

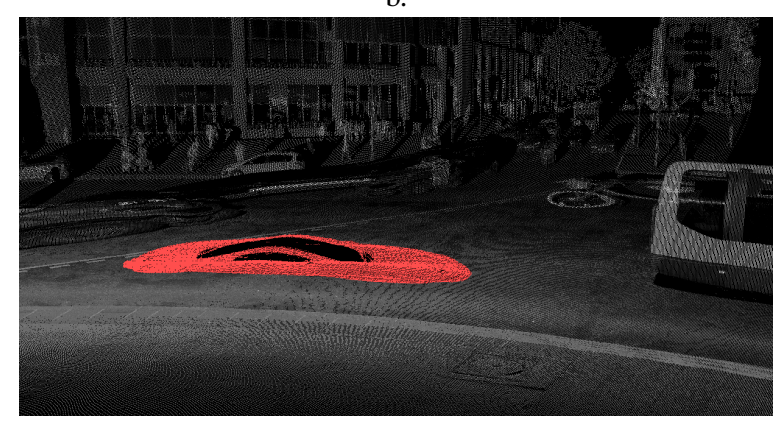

c.

Figure 10. Result of the disocclusion on a car in a dense point cloud. (a) is the original point cloud colorized with the reflectance, (b) is the segmentation of the car highlighted in orange, (c) is the result of the disocclusion.

The test was done on 20 point clouds in which an area was manually removed and then reconstructed. After that, we computed the MAE (Mean Absolute Error) between the ground truth and the reconstruction (where the occlusion was simulated) using both Gaussian disocclusion and our model. We recall that the MAE is expressed as follows:

$$
\operatorname{MAE}\left(u_{1}, u_{2}\right)=\frac{1}{N} \sum_{i, j \in \Omega}\left|u_{1}(i, j)-u_{2}(i, j)\right|
$$

where $u_{1}, u_{2}$ are images defined on $\Omega$ with $N$ pixels. Table 1 sums up the result of our experiment. We can note that our method provides a great improvement compared to the Gaussian disocclusion, with an average MAE lower than $3 \mathrm{~cm}$. This result is largely satisfying as most of the structures to reconstruct were

Table 1. Comparison of the average MAE (Mean Absolute Error) on the reconstruction of occluded areas.

\begin{tabular}{ccc}
\hline & Gaussian & Proposed model \\
\hline Average MAE (meters) & 0.591 & 0.0279 \\
Standard deviation of MAEs & 0.143 & 0.0232 \\
\hline
\end{tabular}

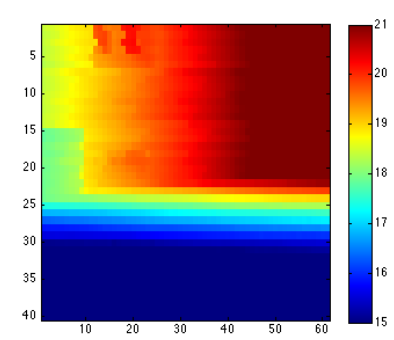

a.

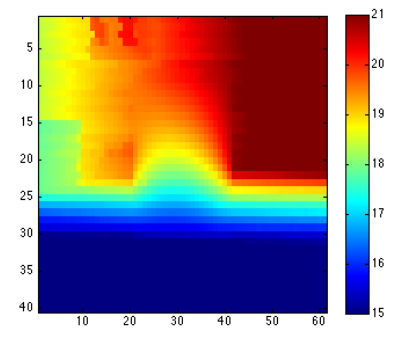

c.

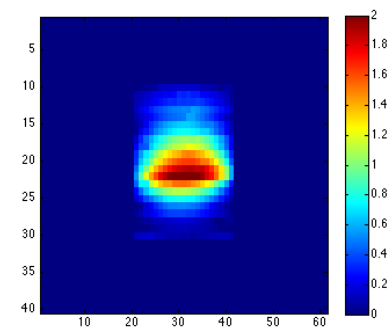

e.

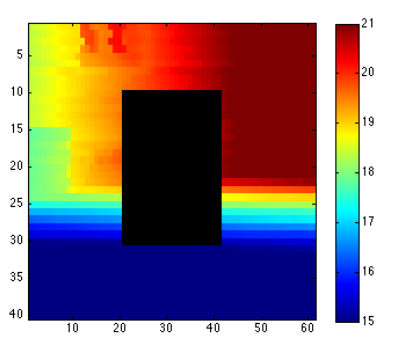

b.

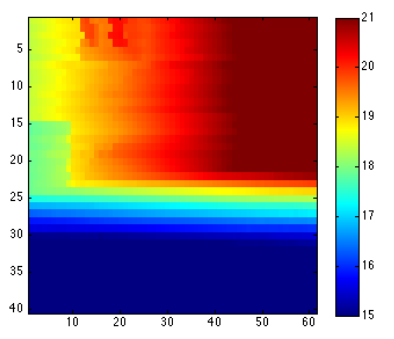

d.

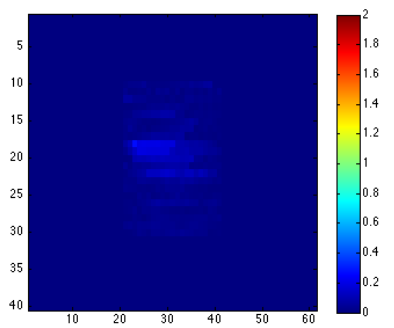

f.
Figure 11. Example of results obtained for the quantitative experiment. (a) is the original point cloud (ground truth), (b) the artificial occlusion in dark, (c) the disocclusion result with the Gaussian diffusion, (d) the disocclusion using our method, (e) the Absolute Difference of the ground truth against the Gaussian diffusion, (f) the Absolute Difference of the ground truth against our method. Scales are given in meters.

situated from 12 to 25 meters away from the sensor.

Figure 11 shows an example of disocclusion following this protocole. The result of our proposed model is visually very plausible whereas the Gaussian diffusion ends up oversmoothing the reconstructed range image which increases the MAE.

\section{CONCLUSION}

In this paper, we have proposed a novel approach for the segmentation and the disocclusion of objects in 3D point clouds acquired using MMS. This model takes advantage of a simplified representation of the point cloud known as a range image. We have also proposed an improvement of a classical imaging technique that takes the nature of the point cloud into account (horizontality prior on the $3 \mathrm{D}$ embedding), leading to better results. The segmentation step can be done in streaming any time a new window is acquired, leading to great speed improvement, constant memory processing and the possibility of online processing during the acquisition. Moreover, our model is designed to work semi-automatically using very few parameters in reasonable computational time. Finally, we have shown that this work performs well in various cases, both on dense and sparse point clouds. 
Considering the range image derived from the sensor topology enabled a simplified formulation of the problem from having to determine an unknown number of $3 \mathrm{D}$ points to estimating only the 1D depth in the ray directions of a fixed set of range image pixels. Beyond simplifying drastically the search space, it also provides directly a reasonable sampling pattern for the reconstructed point set.

Although the average results of the method are more than acceptable, it can underperform in some specific cases. Indeed, the segmentation step first relies on the good extraction of non-ground points, which can be tedious when the data quality is low. Moreover, when the object that needs to be removed from the scene is hiding complex shapes, the disocclusion step can fail recovering all the details of the background and the result ends up being too smooth. This is likely to happen when disoccluding very large objects.

In the future, we will focus on improving the current model to perform better reconstruction by taking into account the neighborhood of the background of the object to remove either by using a variational method or by extending patch-based method.

\section{ACKNOWLEDGEMENT}

J-F. Aujol is a member of Institut Universitaire de France. This work was funded by the ANR GOTMI (ANR-16-CE33-0010-01) grant.

\section{REFERENCES}

Achanta, R., Shaji, A., Smith, K., Lucchi, A., Fua, P. and Süsstrunk, S., 2012. SLIC superpixels compared to state-of-theart superpixel methods. IEEE Trans. on Pattern Analysis and Machine Intelligence.

Becker, J., Stewart, C. and Radke, R. J., 2009. LIDAR inpainting from a single image. In: IEEE Int. Conf. on Computer Vision.

Bertalmio, M., Sapiro, G., Caselles, V. and Ballester, C., 2000. Image inpainting. In: ACM Comp. graphics and interactive techniques.

Bevilacqua, M., Aujol, J.-F., Biasutti, P., Brédif, M. and Bugeau, A., 2017. Joint inpainting of depth and reflectance with visibility estimation. ISPRS Journal of Photogrammetry and Remote Sensing.

Bredies, K., Kunisch, K. and Pock, T., 2010. Total generalized variation. SIAM Journal on Imaging Sciences.

Brédif, M., Vallet, B. and Ferrand, B., 2015. Distributed dimensionality-based rendering of LIDAR point clouds. International Archives of the Photogrammetry, Remote Sensing and Spatial Information Sciences.

Buyssens, P., Daisy, M., Tschumperlé, D. and Lézoray, O., 2015a. Depth-aware patch-based image disocclusion for virtual view synthesis. In: ACM SIGGRAPH.

Buyssens, P., Daisy, M., Tschumperlé, D. and Lézoray, O., 2015b. Exemplar-based inpainting: Technical review and new heuristics for better geometric reconstructions. IEEE Trans. on Image Processing.

Chambolle, A. and Pock, T., 2011. A first-order primal-dual algorithm for convex problems with applications to imaging. Jour. of Math. Imag. and Vis.
Criminisi, A., Pérez, P. and Toyama, K., 2004. Region filling and object removal by exemplar-based image inpainting. IEEE Trans. on Image Processing.

Delon, J., Desolneux, A., Lisani, J.-L. and Petro, A. B., 2007. A nonparametric approach for histogram segmentation. IEEE Trans. on Image Processing.

Demantke, J., Mallet, C., David, N. and Vallet, B., 2011. Dimensionality based scale selection in 3D LIDAR point clouds. International Archives of the Photogrammetry, Remote Sensing and Spatial Information Sciences.

Doria, D. and Radke, R., 2012. Filling large holes in lidar data by inpainting depth gradients. In: Int. Conf. on Pattern Recognition.

El-Halawany, S., Moussa, A., Lichti, D. and El-Sheimy, N., 2011. Detection of road curb from mobile terrestrial laser scanner point cloud. In: Proceedings of the ISPRS Workshop on Laserscanning, Calgary, Canada., Vol. 2931.

Ferstl, D., Reinbacher, C., Ranftl, R., Rüther, M. and Bischof, H., 2013. Image guided depth upsampling using anisotropic total generalized variation. In: IEEE Int. Conf. on Computer Vision.

Geiger, A., Lenz, P., Stiller, C. and Urtasun, R., 2013. Vision meets robotics: The KITTI dataset. International Journal of Robotics Research.

Goulette, F., Nashashibi, F., Abuhadrous, I., Ammoun, S. and Laurgeau, C., 2006. An integrated on-board laser range sensing system for on-the-way city and road modelling. International Archives of the Photogrammetry, Remote Sensing and Spatial Information Sciences.

Hervieu, A. and Soheilian, B., 2013. Semi-automatic road/pavement modeling using mobile laser scanning. ISPRS Annals of the Photogrammetry, Remote Sensing and Spatial Information Sciences.

Hervieu, A., Soheilian, B. and Brédif, M., 2015. Road marking extraction using a MODEL\&DATA-DRIVEN Rj-Mcmc. ISPRS Annals of the Photogrammetry, Remote Sensing and Spatial Information Sciences.

Huang, J. and Menq, C.-H., 2001. Automatic data segmentation for geometric feature extraction from unorganized 3D coordinate points. IEEE Trans. on Robotics and Automation.

Lorenzi, L., Melgani, F. and Mercier, G., 2011. Inpainting strategies for reconstruction of missing data in VHR images. IEEE Trans. on Geoscience and Remote Sensing Letters.

Paparoditis, N., Papelard, J.-P., Cannelle, B., Devaux, A., Soheilian, B., David, N. and Houzay, E., 2012. Stereopolis II: A multi-purpose and multi-sensor 3D mobile mapping system for street visualisation and 3D metrology. Revue française de photogrammétrie et de télédétection.

Papon, J., Abramov, A., Schoeler, M. and Worgotter, F., 2013. Voxel cloud connectivity segmentation-supervoxels for point clouds. In: IEEE Conf. on Computer Vision and Pattern Recognition.

Park, S., Guo, X., Shin, H. and Qin, H., 2005. Shape and appearance repair for incomplete point surfaces. In: IEEE Int. Conf. on Computer Vision, Vol. 2.

Rabbani, T., Van Den Heuvel, F. and Vosselmann, G., 2006. Segmentation of point clouds using smoothness constraint. International Archives of the Photogrammetry, Remote Sensing and Spatial Information Sciences. 
Schnabel, R., Wahl, R. and Klein, R., 2007. RANSAC based out-of-core point-cloud shape detection for city-modeling. Proceedings of Terrestrisches Laserscanning”.

Serna, A. and Marcotegui, B., 2013. Urban accessibility diagnosis from mobile laser scanning data. ISPRS Journal of Photogrammetry and Remote Sensing.

Sharf, A., Alexa, M. and Cohen-Or, D., 2004. Context-based surface completion. ACM Trans. on Graphics.

Weickert, J., 1998. Anisotropic diffusion in image processing. Vol. 1, Teubner Stuttgart.

Weinmann, M., Jutzi, B., Hinz, S. and Mallet, C., 2015. Semantic point cloud interpretation based on optimal neighborhoods, relevant features and efficient classifiers. ISPRS Journal of Photogrammetry and Remote Sensing.

Zhu, X., Zhao, H., Liu, Y., Zhao, Y. and Zha, H., 2010. Segmentation and classification of range image from an intelligent vehicle in urban environment. In: IEEE Trans. on Intelligent Robots and Systems. 